\title{
Clinical and virulence factors related to the 30-day mortality of Klebsiella pneumoniae bacteremia at a tertiary hospital: a case-control study
}

Hiroki Namikawa, Makoto Niki, Mamiko Niki, Koichi Yamada, Kiyotaka Nakaie, Arata Sakiyama, Ken-Ichi Oinuma, Taishi Tsubouchi, Yoshihiro Tochino, Yasuhiko Takemoto, Yukihiro Kaneko, Taichi Shuto, Hiroshi Kakeya

\begin{tabular}{|c|l|}
\hline Citation & European Journal of Clinical Microbiology \& Infectious Diseases. 38(12); 2291-2297 \\
\hline Issue Date & $2019-10-11$ \\
\hline Type & Journal Article \\
\hline Textversion & author \\
\hline Rights & $\begin{array}{l}\text { This is a post-peer-review, pre-copyedit version of an article published in European } \\
\text { Journal of Clinical Microbiology \& Infectious Diseases. The final authenticated } \\
\text { version is available online at: https://doi.org/10.1007/s10096-019-03676-y. } \\
\text { Springer Nature terms of use : } \\
\text { https://www.springer.com/gp/open-access/publication-policies/aam-terms-of-use. }\end{array}$ \\
\hline DOI & $10.1007 /$ s10096-019-03676-y \\
\hline
\end{tabular}

Self-Archiving by Author(s)

Placed on: Osaka City University

Namikawa, H., Niki, M., Niki, M. et al. Clinical and virulence factors related to the 30-day mortality of Klebsiella pneumoniae bacteremia at a tertiary hospital: a case-control study. European Journal of Clinical Microbiology \& Infectious Diseases. 38, 2291-2297. (2019). doi:10.1007/s10096-019-03676-y 
Original article

Clinical and virulence factors related to the 30-day mortality of Klebsiella pneumoniae bacteremia at a tertiary hospital: a case-control study

Hiroki Namikawa ${ }^{1,2}$, Makoto Niki $^{3}$, Mamiko Niki ${ }^{4,5}$, Koichi Yamada ${ }^{1,3,5}$, Kiyotaka Nakaie ${ }^{1,3}$, Arata Sakiyama $^{4}$, Ken-Ichi Oinuma ${ }^{4,5}$, Taishi Tsubouchi ${ }^{4,5}$, Yoshihiro Tochino ${ }^{2}$, Yasuhiko Takemoto $^{2}$, Yukihiro Kaneko ${ }^{4,5}$, Taichi Shuto ${ }^{2}$, Hiroshi Kakeya ${ }^{1,3,5}$

${ }^{1}$ Department of Infection Control Science, Osaka City University, Graduate School of Medicine, 1-4-3, Asahi-machi, Abeno-ku, Osaka 545-8585, Japan

${ }^{2}$ Department of Medical Education and General Practice, Osaka City University, Graduate School of Medicine, 1-4-3, Asahi-machi, Abeno-ku, Osaka 545-8585, Japan

${ }^{3}$ Department of Infection Control and Prevention, Osaka City University Hospital, 1-5-7 Asahi-machi, Abeno-ku, Osaka 545-8586, Japan

${ }^{4}$ Department of Bacteriology, Osaka City University, Graduate School of Medicine, 1-4-3, Asahi-machi, Abeno-ku, Osaka 545-8585, Japan

${ }^{5}$ Research Center for Infectious Disease Sciences (RCIDs), Osaka City University Graduate School of Medicine, 1-4-3, Asahi-machi, Abeno-ku, Osaka 545-8585, Japan

\section{Corresponding author}


Hiroshi Kakeya, MD, PhD

Department of Infection Control Science, Osaka City University, Graduate School of Medicine

1-4-3, Asahi-machi, Abeno-ku, Osaka 545-8585, Japan

Tel: 81-6-6645-3784; Fax: 81-6-6646-6056

E-mail: kakeya@med.osaka-cu.ac.jp

Acknowledgements: This research was supported by the Research Program on Emerging and Re-emerging Infectious Diseases from the Japan Agency Development, AMED [Grant number JP 17fk0108208, 18fk0108052h0002 and 19fk0108094] and JSPS KAKENHI [Grant number 16K09939 and 19K16650]. 


\begin{abstract}
Purpose: Klebsiella pneumoniae bacteremia is a critical clinical presentation that is associated with high mortality. However, extremely few studies have investigated the virulence factors related to mortality of $K$. pneumoniae bacteremia in patients. The present study elucidated clinical and virulence factors associated with the 30-day mortality of $K$. pneumoniae bacteremia at a tertiary hospital.
\end{abstract}

Methods: The medical records of 129 patients with $K$. pneumoniae bacteremia admitted to Osaka City University Hospital between January 2012 and December 2018 were retrospectively reviewed. Patient background characteristics, antimicrobial regimens, and prognosis were evaluated. Additionally, virulence factors were assessed using multiplex polymerase chain reaction to elucidate their association with $K$. pneumoniae.

Results: The 30 -day mortality was $10.9 \%$ in patients with $K$. pneumoniae bacteremia. The male-to-female ratio, age, and underlying disease did not differ between the non-survivor and survivor groups. Multivariate analysis showed that sepsis [odds ratio (OR), 7.46; $p=0.005$ ] and $i u t A(\mathrm{OR}, 4.47 ; p=0.046)$ were independent predictors associated with the 30 -day mortality of $K$. pneumoniae bacteremia.

Conclusion: Despite the relatively low 30-day mortality of patients with $K$. pneumoniae bacteremia, the treatment of those with sepsis and those infected with K. pneumoniae harboring iutA may require careful management for improving their outcomes. 
Keywords: bacteremia; iutA; Klebsiella pneumoniae; mortality; sepsis 


\section{Introduction}

Klebsiella pneumoniae is a common gram-negative pathogen in community- or nosocomial-acquired infections. It causes urinary tract and surgical site infections, pneumonia, and bloodstream infections [1]. Particularly, K. pneumoniae bacteremia is a clinically critical presentation associated with high mortality. Previous studies have indicated that mortality was approximately $16 \%-40 \%$ in patients with K. pneumoniae bacteremia [2-4]. Therefore, elucidating the predictors associated with mortality in K. pneumoniae bacteremia is critical in clinical practice.

A new hypervirulent variant of $K$. pneumoniae has recently emerged, mainly in the Asian Pacific Rim [5]. In recent years, hypervirulent K. pneumoniae (hvKP) infections, which have spread globally, are increasingly recognized as a critical clinical issue worldwide. HvKP can lead to life-threating community-acquired infections including bacteremia, liver abscesses, endophthalmitis, and meningitis [5]. We have previously reported two serious cases of hvKP infection that caused multiple organ abscesses and endophthalmitis [6]. Moreover, hvKP strains are characterized by their distinct sticky phenotype on agar plates. Therefore, this hypermucoviscous phenotype has widely been recognized to be strongly related to its hypervirulence, and the term "hypermucoviscous" has often been used as a synonym of "hypervirulence." However, a recent study suggested that the hypermucoviscosity and hypervirulence were two distinct phenotypes that should not be used as synonyms [7]. The hypervirulence of $K$. pneumoniae should be defined by its genetic 
background and should not solely rely on the bacterial phenotype. In other words, hypermucoviscosity determined by the string test is inadequate to suggest the hypervirulence of the isolated strain. Several studies have reported that the hvKP strain is strongly associated with mortality in in vivo mouse infection models [8,9]. However, studies focusing on the virulence factors related to mortality caused by K. pneumoniae bacteremia in humans are scarce [2].

The present study aimed to investigate clinical variables and virulence factors associated with the 30-day mortality of K. pneumoniae bacteremia at a tertiary hospital.

\section{Materials and Methods}

The medical records of 129 patients with $K$. pneumoniae bacteremia who had been admitted to Osaka City University Hospital between January 2012 and December 2018 were retrospectively reviewed. Patient characteristics including age, sex, underlying disease, clinical features, therapies, and prognosis were evaluated. In cases where K. pneumoniae had been isolated on multiple occasions within the 6-year period in the same patient, only the first episode of $K$. pneumoniae bacteremia was reviewed. The present study was approved by the Ethics Committee of Osaka City University, and the thesis was approved on Mar 22, 2019, with approval number 4299. The need for written informed consent was waived owing to the retrospective nature of the study. 


\section{Definition and source of bacteremia}

Bacteremia was defined as the presence of one or more positive blood cultures in patients with clinical signs of infection such as fever, shaking chills, and sweats, with or without local signs and symptoms. The diagnosis of biliary tract $K$. pneumoniae infection was based on the presence of three or more of the following clinical and diagnostic findings: 1) fever and/or chills; 2) laboratory evidence of an inflammatory response; 3) jaundice or abnormal liver chemistry; 4) biliary dilation or the evidence of an etiology observed on imaging; and 5) $\mathrm{K}$. pneumoniae isolated from a bile specimen. Patients were diagnosed with urinary tract infection (UTI) due to K. pneumoniae in the presence of two or more of the following clinical and diagnostic findings: 1) K. pneumoniae confirmed in a urine specimen, 2) clinical manifestations suggestive of UTI, and 3) imaging findings suggestive of pyelonephritis. The following characteristic symptoms and urinary findings were considered to define UTI: dysuria, suprapubic pain, hematuria, flank pain, costovertebral angle tenderness, nausea or vomiting, and pyuria or bacteriuria [10]. Furthermore, the following imaging findings were considered to support a UTI diagnosis: perinephric stranding, renal swelling, Gerota's fascia thickening, and poor segmental enhancement region [11]. K. pneumoniae pneumonia was diagnosed in the presence of new persistent pulmonary infiltrates, which were not otherwise explained, appearing on chest radiographs along with purulent respiratory secretions and systemic signs of an inflammatory response [12]. Catheter-related bloodstream infection due to $K$. pneumoniae was diagnosed based on one or more of the following clinical and 
diagnostic findings: 1) K. pneumoniae growth was detected in at least one percutaneous blood and catheter-tip culture and 2) K. pneumoniae growth was detected in a blood sample obtained from a catheter hub at least $2 \mathrm{~h}$ before its growth was detected in a peripheral vein blood sample [13].

\section{Assessment of laboratory data}

In patients with a positive initial blood culture, the leukocyte count as well as C-reactive protein and albumin levels were assessed within 2 days of the culture. The 2016 Third International Consensus Definitions for Sepsis and Septic Shock (Sepsis-3) criteria were used in the present study.

\section{Identification of bacteria}

All K. pneumoniae isolates were identified via colony morphologic analysis and Gram staining. Isolate identification, antimicrobial susceptibility, and minimum inhibitory concentrations (MICs) were confirmed using a MicroScan WalkAway-96 SI instrument (Beckman Coulter, Brea, CA, USA). The results were interpreted using the 2018 Clinical and Laboratory Standards Institute (CLSI) criteria. The production of extended-spectrum beta-lactamase (ESBL) was screened by measuring the MICs of cefotaxime, ceftazidime, and aztreonam. Confirmatory testing was performed using an Ambler class C \& ESBL Identification Set (Kanto Chemical, Tokyo, Japan). The production of carbapenemase was 
screened using the sodium mercaptoacetate inhibition test. All plates were incubated at $35^{\circ} \mathrm{C}$ for $24 \mathrm{~h}$.

\section{Antimicrobial therapies}

The attending physician determined the appropriate initial antimicrobial therapy. Antimicrobial therapy administered within 5 days after bacteremia onset was defined as empirical therapy, whereas that administered thereafter as definitive therapy. Appropriate empirical or definitive antibiotic therapy was defined as that matching the in vitro susceptibility results according to the CLSI criteria [14].

\section{Virulence factors}

The following nine virulence genes were assessed using multiplex polymerase chain reaction (PCR), as previously described [15]: $m a g A$ (specific to K1 capsule serotype) and $r m p A$ for mucoviscosity; ent $B, y b t S, k f u$, and iutA for siderophore pathway; mrkD for adhesins; allS for allantoin metabolism; and wzi for the specific K2 capsular serotype. Primers used for multiplex PCR are listed in Table 1.

\section{Statistical analysis}

Patient characteristics, blood test data, therapies, and bacterial characteristics were compared between the survivor and non-survivor groups. The Mann-Whitney $U$ and chi-square tests 
were used for the univariate comparison of categorical data. To determine the independent predictors of the 30-day mortality of K. pneumoniae bacteremia, variables with a $p$ value $<0.1$ in the univariate analyses were considered for inclusion in the multivariate logistic regressions using EZR (Saitama Medical Center, Jichi Medical University, Saitama, Japan), a graphical interface for R (The R Foundation for Statistical Computing, Vienna, Austria, version 3.5.3). EZR is a modified version of $\mathrm{R}$ commander (version 2.4-0) that includes the statistical functions frequently used in biostatistics. A $p$ value of $<0.05$ was considered statistically significant.

\section{Results}

\section{Clinical characteristics and laboratory findings}

The clinical characteristics and laboratory findings of the non-survivors and survivors of $K$. pneumoniae bacteremia are summarized in Table 2 . The 30 -day mortality in patients with $K$. pneumoniae bacteremia was $10.9 \%$. The non-survivor group $(\mathrm{n}=14)$ included 8 males and 6 females, with a mean age of 66.2 years. The survivor group $(n=115)$ included 72 males and 43 females, with a mean age of 66.5 years. The non-survivor group included $8(61.5 \%), 3$ (23.1\%), and $5(35.7 \%)$ patients with malignancy, diabetes mellitus, and nosocomial-acquired bacteremia, respectively. Conversely, the survivor group included 72 (62.6\%), 40 (34.8\%), and $62(53.9 \%)$ patients with malignancy, diabetes mellitus, and nosocomial-acquired bacteremia, respectively. The Charlson score did not differ between the non-survivor and 
survivor groups $(4.1 \pm 2.9$ vs. $3.4 \pm 2.6, p=0.37)$. However, sepsis was significantly more frequent among patients in the non-survivor group than those in the survivor group $(78.6 \% \mathrm{vs}$. $26.1 \%, p<0.001)$

\section{Therapies}

Table 3 summarizes the empirical and definitive therapies for patients with K. pneumoniae bacteremia in the non-survivor and survivor groups. The most frequently used antibiotics for both empirical and definitive therapy were carbapenems in both the non-survivor and survivor groups (empirical: $64.3 \%$ and $33.0 \%$, respectively; definitive: $28.6 \%$ and $24.8 \%$, respectively). The rates of appropriate empirical or definitive therapy did not differ between both the non-survivor and survivor groups (empirical: 100\% and 94.8\%, respectively, $p=$ 0.84; definitive: $100 \%$ and $96.5 \%$, respectively, $p=1.00$ ).

\section{Bacterial characteristics}

Bacterial characteristics observed in the non-survivor and survivor groups are shown in Table 4. Although $10(8.7 \%)$ K. pneumoniae isolates in the survivor group were ESBL-producing strains, none of the isolates in the non-survivor group were ESBL-producing strains. Notably, the frequency of the iutA was higher in the non-survivor group than the survivor group $(35.7 \%$ vs. $9.6 \%, p=0.02)$, whereas the frequency of other virulence genes was not significantly different between the two groups. 
Prognostic factors

Multivariate analysis (Table 5) revealed that sepsis [odds ratio (OR), 7.46; $p=0.005$ ] and iutA (OR, 4.47; $p=0.046)$ were independent predictors of the 30-day mortality of $K$. pneumoniae bacteremia.

\section{Discussion}

Our single-center, case-control study including 129 patients with K. pneumoniae bacteremia revealed that the 30 -day mortality in patients with $K$. pneumoniae bacteremia was $10.9 \%$, which was lower than that previously reported [2-4]. Second, it was suggested that sepsis and iutA are independent predictors of the 30-day mortality of $K$. pneumoniae bacteremia. The relatively lower 30 -day mortality of $10.9 \%$ compared with previous studies might be attributable to several factors. First, most previous studies have reported that nosocomial $K$. pneumoniae infections were associated with a higher mortality compared with community-acquired K. pneumoniae infections [16-18]. Furthermore, nosocomial infections due to K. pneumoniae bacteremia were more frequent than community-acquired infections due to $K$. pneumoniae bacteremia, ranging from $55 \%$ to $92 \%$ [4,16-18]. On the other hand, the rate of nosocomial K. pneumoniae infections in the present study was $52 \%$, which was lower than that previously reported; this lower rate might be associated with the lower mortality observed in the present study. Second, a previous study has reported that 
administering the appropriate empirical antibiotic therapy was an important predictor of patient outcomes [19]. Furthermore, previous reports have demonstrated that the rate of appropriate antibiotic therapy administered for K. pneumoniae bacteremia varied from $46 \%$ to $85 \%[2,4,17,19]$. In contrast, the rate of appropriate empirical therapy in the present study was $95 \%$, which was prominently higher than the previously reported rates. The frequency of resistant bacteria might therefore contribute to the differences in mortality. Therefore, these findings support that the 30-day mortality in the present study was lower than that reported by previous studies.

K. pneumoniae is one of the most frequently isolated bacteria from patients with sepsis and is known to harbor various virulence factors [20]. The capsule of $K$. pneumoniae has multiple different functions: it prevents phagocytosis and the functioning of antimicrobial peptides, blocks complement-mediated lysis and opsonization, and averts the inflammatory response. Considering the diverse roles of the capsule, K. pneumoniae infections are associated with a potentially severe clinical presentation. Moreover, $K$. pneumoniae endotoxin, which is produced by other gram-negative bacteria as well, induces cytokines and leads to organ dysfunction and sepsis [21]. These findings suggest that sepsis is associated with an increased risk of mortality in patients with $K$. pneumoniae bacteremia.

Bacteria have developed the iron acquisition tools termed as siderophores to compete with the host [22]. In addition to its iron acquisition function, siderophores can modulate host immune responses [23], enhance bacterial dissemination [24], and regulate the virulence 
factor production [25]. Aerobactin, a citrate-hydroxamate siderophore, is produced by several pathogenic gram-negative bacteria to help iron assimilation. Aerobactin has increasingly been recognized for its key role in mediating the enhanced virulence of hvKP. Recently, Russo et al. showed that aerobactin is a key factor that distinguished hvKP from non-hvKP strains [26]. Aerobactin gene, along with $r m p A$, was frequently isolated from $K$. pneumoniae bacteremic strains that caused primary liver abscess [27]. Moreover, aerobactin is identified as an important virulence factor associated with mortality in an in vivo mouse infection model [9]. $i u t A$ encodes for the outer-membrane receptor for ferric aerobactin. Vargas et al. reported that iutA promotes biofilm formation [28]. Moreover, Tang et al. showed that iutA is an independent pathogenicity factor for abscess formation [29]. A few clinical studies have reported that $i u t A$ is associated with a high pathogenicity of $K$. pneumoniae [30,31]. Together, these findings suggest that iutA is associated with an increased risk of mortality in patients with K. pneumoniae bacteremia. However, we did not examine the other siderophore-related genes, such as $i u c A$ or $i r o B$. In the future, these genes should be analyzed to determine their association with the mortality of $K$. pneumoniae bacteremia.

The present study has several limitations. First, the study population was relatively small, and the study was conducted in one tertiary hospital only; therefore, there was selection bias. Future studies including more patients with $K$. pneumoniae bacteremia in both community and tertiary hospital settings are required to address this limitation. Second, we examined only nine virulence genes in $K$. pneumoniae, and additional virulence genes of $K$. 
pneumoniae that have been reported to date should be analyzed to determine their association with the mortality of $K$. pneumoniae bacteremia. Third, the primary aim of this retrospective case-control study was to elucidate the predictive factors of mortality caused by $K$. pneumoniae bacteremia; however, prospective studies are required to further clarify these factors.

In conclusion, the present study demonstrated that the 30-day mortality in patients with $K$. pneumoniae bacteremia was relatively low at $10.9 \%$. Sepsis and iutA may be independent predictors of the 30-day mortality of $K$. pneumoniae bacteremia. Therefore, the treatment of patients with bacteremia caused by K. pneumoniae in the state of sepsis and of those infected with $K$. pneumoniae harboring iutA may require careful management for improving their outcomes.

Conflicts of interest: The authors declare that there are no conflicts of interest.

Funding: This research was supported by the Research Program on Emerging and Re-emerging Infectious Diseases from the Japan Agency Development, AMED [Grant number JP 17fk0108208, 18fk0108052h0002 and 19fk0108094] and JSPS KAKENHI [Grant number 16K09939 and 19K16650].

Ethical approval: The study was approved by the Ethics Committee of Osaka City University, and the thesis was approved on Mar 22, 2019, with approval number 4299. Informed consent: Not applicable to this study. 


\section{References}

1. Magill SS, Edwards JR, Bamberg et al (2014)

Multistate point-prevalence survey of health care-associated infections. N Engl J Med 370(13):1198-1208

2. Kim D, Park BY, Choi MH et al (2019) Antimicrobial resistance and virulence factors of Klebsiella pneumoniae affecting 30 day mortality in patients with bloodstream infection. J Antimicrob Chemother 74(1):190-199

3. Li L, Huang H (2017) Risk factors of mortality in bloodstream infections caused by Klebsiella pneumonia: A single-center retrospective study in China. Medicine (Baltimore) 96(35):e7924

4. Li J, Ren J, Wang W et al (2018) Risk factors and clinical outcomes of hypervirulent Klebsiella pneumoniae induced bloodstream infections. Eur J Clin Microbiol Infect Dis 37(4):679-689

5. Shon AS, Bajwa RP, Russo TA (2013) Hypervirulent (hypermucoviscous) Klebsiella pneumoniae: a new and dangerous breed. Virulence 4(2):107-118

6. Namikawa H, Yamada K, Fujimoto H et al (2016) 
Two unusual cases of successful treatment of hypermucoviscous Klebsiella pneumoniae invasive syndrome. BMC Infect Dis 16(1):680

7. Catalán-Nájera JC, Garza-Ramos U, Barrios-Camacho H. (2017) Hypervirulence and hypermucoviscosity: Two different but complementary Klebsiella spp. phenotypes?

Virulence 8(7):1111-1123

8. Lery LM, Frangeul L, Tomas A et al (2014) Comparative analysis of Klebsiella pneumoniae genomes identifies a phospholipase $\mathrm{D}$ family protein as a novel virulence factor. BMC Biol 12:41

9. Russo TA, Olson R, MacDonald U et al (2015) Aerobactin, but not yersiniabactin, salmochelin, or enterobactin, enables the growth/survival of hypervirulent (hypermucoviscous) Kleb siella pneumoniae ex vivo and in vivo. Infect Immun 83(8):3325-3333

10. Hooton TM (2012) Clinical practice. Uncomplicated urinary tract infection. N Engl J Med 366(11):1028-1037

11. Hammond NA, Nikolaidis P, Miller FH (2012) Infectious and inflammatory diseases 
of the kidney. Radiol Clin North Am 50(2):259-270

12. Magret M, Lisboa T, Martin-Loeches I et al (2011) Bacteremia is an independent risk factor for mortality in nosocomial pneumonia: a prospective and observational multicenter study. Crit Care 15(1):R62

13. Mermel LA, Allon M, Bouza E et al (2009) Clinical practice guidelines for the diagnosis and management of intravascular catheter-related infection: 2009 Update by the Infectious Diseases Society of America. Clin Infect Dis 49(1):1-45

14. Micek ST, Welch EC, Khan J et al (2010) Empiric combination antibiotic therapy is associated with improved outcome against sepsis due to Gram-negative bacteria: a retrospective analysis. Antimicrob Agents Chemother 54(5):1742-1748.

15. Compain F, Babosan A, Brisse S et al (2014) Multiplex PCR for detection of seven virulence factors and K1/K2 capsular serotypes of Klebsiella pneumoniae. J Clin Microbiol 52(12):4377-4380

16. Meatherall BL, Gregson D, Ross T et al (2009) Incidence, risk factors, and outcomes of Klebsiella pneumoniae bacteremia. Am J Med 122(9):866-873 
17. Tian L, Tan R, Chen Y et al (2016) Epidemiology of Klebsiella pneumoniae bloodstream infections in a teaching hospital: factors related to the carbapenem resistance and patient mortality. Antimicrob Resist Infect Control 5:48

18. Pau CK, Ma FF, Ip M et al (2015) Characteristics and outcomes of Klebsiella pneumoniae bacteraemia in Hong Kong. Infect Dis (Lond) 47(5):283-288

19. Man MY, Shum HP, Chan YH et al (2017)

Clinical predictors and outcomes of Klebsiella pneumoniae bacteraemia in a regional hospital in Hong Kong. J Hosp Infect 97(1):35-41

20. Paczosa MK, Mecsas J (2016) Klebsiella pneumoniae: Going on the Offense with a Strong Defense. Microbiol Mol Biol Rev 80(3):629-661

21. Angus DC, van der Poll T (2013) Severe sepsis and septic shock. N Engl J Med 369(9):840-851

22. Miethke M, Marahiel MA (2007) Siderophore-based iron acquisition and pathogen control. Microbiol Mol Biol Rev 71(3):413-451

23. Saha P, Yeoh BS, Olvera RA et al (2017) Bacterial Siderophores Hijack Neutrophil 
Functions. J Immunol 198(11):4293-4303

24. Holden VI, Breen P, Houle S et al (2016) Klebsiella pneumoniae Siderophores Induce Inflammation, Bacterial Dissemination, and HIF-1 $\alpha$ Stabilization during Pneumonia. MBio 7(5). pii: e01397-16

25. Lamont IL, Beare PA, Ochsner U et al (2002)

Siderophore-mediated signaling regulates virulence factor production in Pseudomonasaeruginosa. Proc Natl Acad Sci U S A 99(10):7072-7077

26. Russo TA, Olson R, Fang CT et al (2018) Identification of Biomarkers for Differentiation of Hypervirulent Klebsiella pneumoniae from Classical K. pneumoniae.

J Clin Microbiol 56(9). pii: e00776-18

27. Tan TY, Ong M, Cheng Y et al (2019) Hypermucoviscosity, rmpA, and aerobactin are associated with community-acquired Klebsiella pneumoniae bacteremic isolates causing liver abscess in Singapore. J Microbiol Immunol Infect 52(1):30-34

28. Vargas JM, Moreno Mochi MP, Nuñez JM et al (2019) Virulence 
factors and clinical patterns of multiple-clone hypermucoviscous KPC-2

producing K. pneumoniae. Heliyon 5(6):e01829.

29. Tang HL, Chiang MK, Liou WJ et al (2010) Correlation between Klebsiella

pneumoniae carrying pLVPK-derived loci and abscess formation. Eur J Clin Microbiol Infect Dis 29(6):689-698.

30. Gu D, Dong N, Zheng Z et al (2018)

A fatal outbreak of ST11 carbapenem-resistant hypervirulent Klebsiella pneumoniae in a Chinese hospital: a molecular epidemiological study. Lancet Infect Dis 18(1):37-46.

31. Zhan L, Wang S, Guo Y et al (2017) Outbreak by Hypermucoviscous Klebsiella pneumoniae ST11 Isolates with CarbapenemResistance in a Tertiary Hospital in China. Front Cell Infect Microbiol 7:182

32. Fang CT, Chuang YP, Shun CT et al (2004) A novel virulence gene in Klebsiella pneumoniae strains causing primary liver abscess and septic metastatic complications. $\mathrm{J}$ Exp Med 199(5):697-705

33. Yu WL, Fung CP, Ko WC et al (2007) Polymerase chain 
reaction analysis for detecting capsule serotypes $\mathrm{K} 1$ and $\mathrm{K} 2$ of Klebsiella pneumoniae causing abscesses of the liver and other sites. J Infect Dis 195(8):1235-1236; author reply 1236. 
Table 1. Primers used for multiplex PCR

\begin{tabular}{llll}
\hline Primer & Target gene & Sequence $\left(5^{\prime} \rightarrow 3^{\prime}\right)$ & Reference \\
\hline magA-F & $m a g A$ & GGTGCTCTTTACATCATTGC & 32 \\
magA-R & $m a g A$ & GCAATGGCCATTTGCGTTAG & 1 \\
rmpA-F & $r m p A$ & CATAAGAGTATTGGTTGACAG & 1 \\
rmpA-R & $r m p A$ & CTTGCATGAGCCATCTTTCA & 1 \\
entB-F & $e n t B$ & GTCAACTGGGCCTTTGAGCCGTC & 1 \\
entB-R & $e n t B$ & TATGGGCGTAAACGCCGGTGAT & 1 \\
ybtS-F & $y b t S$ & GACGGAAACAGCACGGTAAA & 1 \\
ybtS-R & $y b t S$ & GAGCATAATAAGGCGAAAGA & 1 \\
kfu-F & $k f u$ & GGCCTTTGTCCAGAGCTACG & 1 \\
kfu-R & $k f u$ & GGGTCTGGCGCAGAGTATGC & 1 \\
iutA-F & $i u t A$ & &
\end{tabular}




\begin{tabular}{llll} 
mrkD-F & $m r k D$ & AAGCTATCGCTGTACTTCCGGCA & 1 \\
mrkD-R & $m r k D$ & GGCGTTGGCGCTCAGATAGG & 1 \\
allS-F & alls & CATTACGCACCTTTGTCAGC & 1 \\
allS-R & $a l l S$ & GAATGTGTCGGCGATCAGCTT & 1 \\
wzi-F & $w z i$ & CAACCATGGTGGTCGATTAG & 33 \\
wzi-R & $w z i$ & TGGTAGCCATATCCCTTTGG & 33 \\
\hline
\end{tabular}


Table 2. Clinical characteristics and laboratory findings of non-survivor and survivor patients with K. pneumoniae bacteremia

\begin{tabular}{|c|c|c|c|}
\hline Variables & Non-survivors $(\mathrm{n}=14)$ & Survivors $(n=115)$ & $p$ value \\
\hline Age $(\text { years })^{a}$ & $66.2 \pm 11.1$ & $66.5 \pm 12.9$ & $0.84^{b}$ \\
\hline Male & $8(57.1 \%)$ & $72(62.6 \%)$ & $0.92^{\mathrm{c}}$ \\
\hline \multicolumn{4}{|l|}{ Underlying disease $^{\mathrm{d}}$} \\
\hline Malignancy & $8(61.5 \%)$ & $72(62.6 \%)$ & $1.00^{\mathrm{c}}$ \\
\hline Immunosuppressive drug or corticosteroid use & $1(7.7 \%)$ & $19(16.5 \%)$ & $0.67^{\mathrm{c}}$ \\
\hline Diabetes mellitus & $3(23.1 \%)$ & $40(34.8 \%)$ & $0.59^{c}$ \\
\hline Chronic obstructive pulmonary disease & $3(23.1 \%)$ & $12(10.4 \%)$ & $0.37^{\mathrm{c}}$ \\
\hline Chronic kidney disease & $2(15.4 \%)$ & $24(20.9 \%)$ & $0.92^{\mathrm{c}}$ \\
\hline Lower gastrointestinal disease & $0(0 \%)$ & $7(6.1 \%)$ & $0.79^{c}$ \\
\hline Charlson score ${ }^{\mathrm{a}, \mathrm{d}}$ & $4.1 \pm 2.9$ & $3.4 \pm 2.6$ & $0.37^{b}$ \\
\hline Use of antibiotics prior to isolation d,e & $6(46.2 \%)$ & $60(52.2 \%)$ & $0.91^{\mathrm{c}}$ \\
\hline Leukocyte count $\geq 12000(/ \mu \mathrm{L})$ & $8(57.1 \%)$ & $41(35.7 \%)$ & $0.20^{\mathrm{c}}$ \\
\hline C-reactive protein $\geq 10(\mathrm{mg} / \mathrm{dL})$ & $10(71.4 \%)$ & $53(46.1 \%)$ & $0.09^{\mathrm{c}}$ \\
\hline Albumin $\leq 2.5(\mathrm{~g} / \mathrm{dL})$ & $7(50.0 \%)$ & $27(23.5 \%)$ & $0.07^{\mathrm{c}}$ \\
\hline Nosocomial infection & $5(35.7 \%)$ & $62(53.9 \%)$ & $0.32^{c}$ \\
\hline Operation within 30 days $^{\mathrm{d}}$ & $1(7.7 \%)$ & $11(9.6 \%)$ & $1.00^{\mathrm{c}}$ \\
\hline Hospitalization within 90 days $^{\mathrm{d}}$ & $4(30.8 \%)$ & $48(41.7 \%)$ & $0.64^{\mathrm{c}}$ \\
\hline \multicolumn{4}{|l|}{ Infection site } \\
\hline Biliary tract & $5(35.7 \%)$ & $37(32.2 \%)$ & $1.00^{\mathrm{c}}$ \\
\hline Urinary tract & $1(7.1 \%)$ & $20(17.4 \%)$ & $0.55^{\mathrm{c}}$ \\
\hline Lung & $3(21.4 \%)$ & $9(7.8 \%)$ & $0.24^{\mathrm{c}}$ \\
\hline Intravascular device & $0(0 \%)$ & $6(5.2 \%)$ & $0.84^{c}$ \\
\hline Others & $0(0 \%)$ & $4(3.5 \%)$ & $1.00^{\mathrm{c}}$ \\
\hline Unknown & $5(29.2 \%)$ & $38(33.0 \%)$ & $1.00^{\mathrm{c}}$ \\
\hline
\end{tabular}




\begin{tabular}{clll} 
Abscess $^{\mathrm{f}}$ & $2(16.7 \%)$ & $11(9.6 \%)$ & $0.79^{\mathrm{c}}$ \\
Multiple & $1(7.1 \%)$ & $1(0.87 \%)$ & $0.52^{\mathrm{c}}$ \\
Sepsis & $11(78.6 \%)$ & $30(26.1 \%)$ & $<0.001^{\mathrm{c}}$ \\
\hline
\end{tabular}

${ }^{a}$ Data are presented as means \pm standard deviation

${ }^{\mathrm{b}}$ Mann-Whitney U test

c Chi-square test

${ }^{\mathrm{d}}$ Details were not known for one patient in the non-survivor group

e 60 days prior to isolation

${ }^{\mathrm{f}}$ Details were not known for two patients in the non-survivor group

Table 3. Empirical and definitive therapies in the non-survivor and survivor groups of patients with K. pneumoniae bacteremia

\begin{tabular}{|c|c|c|c|c|}
\hline \multirow[t]{2}{*}{ Variables } & \multicolumn{2}{|c|}{ Empirical therapy } & \multicolumn{2}{|c|}{ Definitive therapy } \\
\hline & Non-survivors $(\mathrm{n}=14)$ & Survivors $(\mathrm{n}=115)$ & Non-survivors $(\mathrm{n}=7)^{\mathrm{a}}$ & Survivors $(\mathrm{n}=113)^{\mathrm{b}}$ \\
\hline Carbapenems & $9(64.3 \%)$ & $38(33.0 \%)$ & $2(28.6 \%)$ & $28(24.8 \%)$ \\
\hline Tazobactam/Piperacillin & $3(21.4 \%)$ & $20(17.4 \%)$ & $0(0 \%)$ & $19(16.8 \%)$ \\
\hline Quinolones & $0(0 \%)$ & $7(6.1 \%)$ & $1(14.3 \%)$ & $11(9.7 \%)$ \\
\hline Fourth-generation cephalosporins & $1(7.1 \%)$ & $11(9.6 \%)$ & $0(0 \%)$ & $9(8.0 \%)$ \\
\hline Third-generation cephalosporins & $1(7.1 \%)$ & $20(17.4 \%)$ & $1(14.3 \%)$ & $14(12.4 \%)$ \\
\hline Sulbactam/ampicillin & $0(0 \%)$ & $8(7.0 \%)$ & $2(28.6 \%)$ & $13(11.5 \%)$ \\
\hline Second-generation cephalosporins & $0(0 \%)$ & $9(7.8 \%)$ & $0(0 \%)$ & $7(6.2 \%)$ \\
\hline First-generation cephalosporins & $0(0 \%)$ & $1(1.7 \%)$ & $1(14.3 \%)$ & $12(10.6 \%)$ \\
\hline Appropriate therapy & $14(100 \%)$ & $109(94.8 \%)$ & $7(100.0 \%)$ & $109(96.5 \%)$ \\
\hline
\end{tabular}

${ }^{a}$ Seven patients died before definitive therapy.

${ }^{\mathrm{b}}$ One patient was transferred to a different hospital, and one patient discontinued the treatment before definitive therapy. 
Table 4. Bacterial characteristics of the non-survivor and survivor groups of patients with K. pneumoniae bacteremia

\begin{tabular}{|c|c|c|c|}
\hline Variables & Non-survivors $(\mathrm{n}=14)$ & Survivors $(\mathrm{n}=115)$ & $p$ value \\
\hline \multicolumn{4}{|l|}{ Beta-lactamase production } \\
\hline ESBL & $0(0 \%)$ & $10(8.7 \%)$ & 0.60 \\
\hline $\mathrm{AmpC}$ & $0(0 \%)$ & $0(0 \%)$ & NA \\
\hline carbapenemase & $0(0 \%)$ & $1(0.9 \%)$ & 1.00 \\
\hline \multicolumn{4}{|l|}{ Virulence gene } \\
\hline \multicolumn{4}{|l|}{ mucoviscosity } \\
\hline $\operatorname{mag} A$ (specific to $\mathrm{K} 1$ capsule serotype) & $5(35.7 \%)$ & $56(48.7 \%)$ & 0.53 \\
\hline$r m p A$ & $4(28.6 \%)$ & $13(11.3 \%)$ & 0.17 \\
\hline \multicolumn{4}{|l|}{ siderophore } \\
\hline entB & $14(100.0 \%)$ & $112(97.4 \%)$ & 1.00 \\
\hline$y b t S$ & $3(21.4 \%)$ & $35(30.4 \%)$ & 0.70 \\
\hline$k f u$ & $4(28.6 \%)$ & $35(30.4 \%)$ & 1.00 \\
\hline iutA & $5(35.7 \%)$ & $11(9.6 \%)$ & 0.02 \\
\hline \multicolumn{4}{|l|}{ adhesin } \\
\hline$m r k D$ & $14(100.0 \%)$ & $112(97.4 \%)$ & 1.00 \\
\hline \multicolumn{4}{|l|}{ allantoin metabolism } \\
\hline allS & $1(7.1 \%)$ & $5(4.3 \%)$ & 1.00 \\
\hline \multicolumn{4}{|l|}{ other } \\
\hline$w z i$ (specific to K2 capsule serotype) & $3(21.4 \%)$ & $10(8.7 \%)$ & 0.31 \\
\hline
\end{tabular}

${ }^{\mathrm{a}}$ Chi-square test

ESBL, extended-spectrum beta-lactamase; NA, not available 
Table 5. Multivariate analysis of predictors associated with the 30-day mortality of K. pneumoniae bacteremia

\begin{tabular}{lcc}
\hline Predictor & OR $(95 \% \mathrm{CI})$ & $p$ value \\
\hline C-reactive protein $\geq 10(\mathrm{mg} / \mathrm{dL})$ & $1.54(0.39-6.03)$ & 0.53 \\
Albumin $\leq 2.5(\mathrm{~g} / \mathrm{dL})$ & $2.60(0.70-9.64)$ & 0.15 \\
Sepsis & $7.46(1.85-30.1)$ & 0.005 \\
iutA & $4.47(1.03-19.5)$ & 0.046 \\
\hline
\end{tabular}

CI, confidence interval; OR, odds ratio 